\title{
NEU at WNUT-2020 Task 2: Data Augmentation To Tell BERT That Death Is Not Necessarily Informative
}

\author{
Kumud Chauhan \\ Northeastern University \\ chauhan.ku@northeastern.edu
}

\begin{abstract}
Millions of people around the world are sharing COVID-19 related information on social media platforms. Since not all the information shared on the social media is useful, a machine learning system to identify informative posts can help users in finding relevant information. In this paper, we present a BERT classifier system for W-NUT2020 Shared Task 2: Identification of Informative COVID-19 English Tweets. Further, we show that BERT exploits some easy signals to identify informative tweets, and adding simple patterns to uninformative tweets drastically degrades BERT performance. In particular, simply adding "10 deaths" to tweets in dev set, reduces BERT F1score from 92.63 to 7.28 . We also propose a simple data augmentation technique that helps in improving the robustness and generalization ability of the BERT classifier.
\end{abstract}

\section{Introduction}

COVID-19 pandemic as well as COVID-19 related information both are spreading across the world rapidly. Easy access to the internet made the consumption and sharing of information much faster. Millions of people are sharing COVID-19 related information using social media channels such as Facebook, Twitter. Social media is divided over several issues related to masks, social distancing, COVID-19 testing, etc. Unfortunately, the response to the coronavirus has been often determined by people's ideology instead of health officials' guidelines. One challenge with massive information available on social media is to separate useful COVID-19 related information from the noise. As a testimony, the director of WHO in Munich Security conference said "We're not just fighting an epidemic; we're fighting an infodemic"1.

\footnotetext{
${ }^{1}$ https://www. who. int/dg/speeches/ detail/munich-security-conference
}

NLP community have taken multiple initiatives to fight this infodemic including misinformation identification (Shahi et al., 2020), finding answers to COVID related questions (Esteva et al., 2020).

In this paper, we present our system in the $\mathrm{W}$ NUT 2020 Shared Task 2: Identification of Informative COVID-19 English Tweets (Nguyen et al., 2020). We use transformer (Vaswani et al., 2017) based models such as BERT (Devlin et al., 2018) to classify COVID-19 tweets into informative and uninformative category. We observe that BERT heavily relies on death-related information to identify informative tweets, and can be easily fooled by adding a simple death count information to any uninformative tweet. Further, to improve robustness of the model, we propose a targeted data augmentation technique. Our contributions are as follows:

- We show that while BERT based models perform well on the shared task dataset, their performance falls drastically if we add deathrelated information to uninformative tweets.

- We propose a simple data augmentation method that aims at replacing the most discriminative words with other words to reduce the model's heavy reliance on specific words.

\section{Task Description}

The goal of the shared task 2 is to classify English tweets into INFORMATIVE or UNINFORMATIVE category. INFORMATIVE tweets provide information about recovered, suspected, confirmed, and death cases as well as location or travel history of the cases. The dataset consists of 10,000 COVID English Tweets, including 4719 Tweets labeled as INFORMATIVE and 5281 Tweets labeled as UNINFORMATIVE. Table 1 shows the descriptive statistics of the train, dev, and test partition of the dataset. 


\begin{tabular}{lrl}
\hline Split & Informative & Uninformative \\
\hline Train & 3303 & 3697 \\
Dev & 472 & 528 \\
Test & 944 & 1056 \\
\hline
\end{tabular}

Table 1: Train, Dev and Testset statistics

\section{Method}

The task is formulated as a binary classification task. Since transformers based models provide state of the art performance on the text classification task, we use a pre-trained BERT as a classifier.

\subsection{Classifiers}

For the classification task, we consider two different variants of the BERT model. Our first model is pre-trained bert-large-uncased BERT model (Devlin et al., 2018). The second model is COVIDTwitter-BERT (CT-BERT) which is a transformerbased model pre-trained on a large corpus of Twitter messages on the topic of COVID-19. (Müller et al., 2020). Note that both models are similar in size and use the same base architecture. The only difference is in terms of the data used for pretraining. While bert-large-uncased is trained on Wikipedia and Books corpus, CT-BERT is trained on $22.5 \mathrm{M}$ tweets corpus.

\subsection{BERT Exploits Easy Clues For Classification}

Machine Learning systems can perform well by relying on heuristics that are effective for frequent example types but break down in more challenging cases. (McCoy et al., 2019; Niven and Kao, 2019). In our exploratory experiments, we found that the BERT classifier heavily relies on some easy clues such as the presence of reported deaths related terms such as "deaths", "died" to identify INFORMATIVE tweets. For example, BERT model classify the following uninformative tweet from validation set (1248314498747895813) as informative.

Data from National Records of Scotland shows that in the last full week, registered deaths from all causes across Scotland was 60\% higher than the five-year average at 1,741 deaths compared with the average of 1,098." Most of that will be \#COVID19? HTTPURL

The above tweet contains death count related information, model fails to recognize it as uninformative tweet. This leads us to experiment with adding such signals to UNINFORMATIVE tweets, to our surprise, it results in a huge drop in classification performance. For example, simply adding " 10 deaths" to all tweets in the dev set, reduces BERT F1-score from 92.63 to 7.28 .

Since such models can break for any uninformative tweet which contains variants of reported deaths, it raises questions about models' robustness and usefulness in a real-world application. In this paper, we explore data augmentation as a method to improve the robustness of such models.

\subsection{Data Augmentation To Improve Robustness And Generalization}

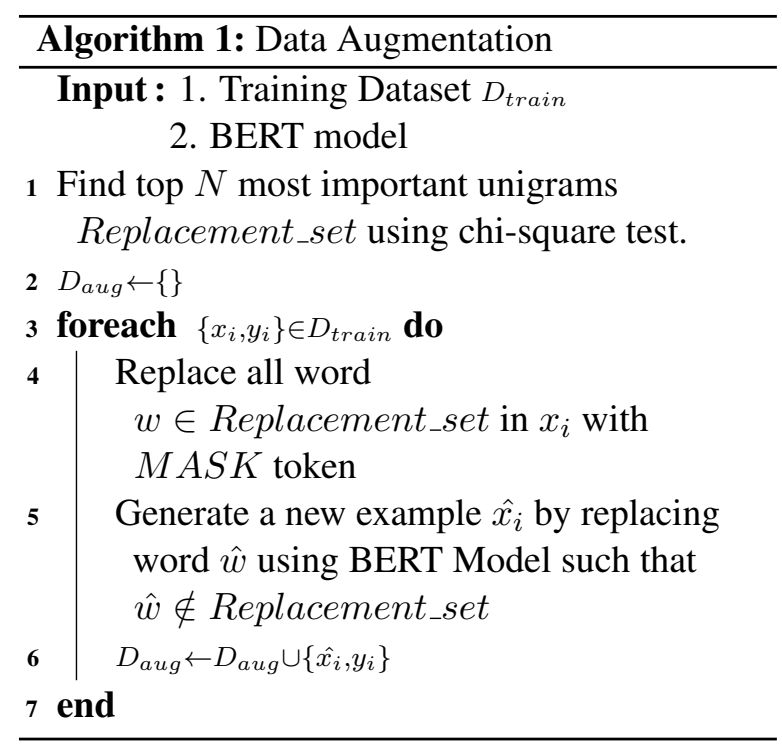

Data Augmentation have been extensively used to improve classification performance in low-data regime (Wu et al., 2019; Kumar et al., 2019; Wei and Zou, 2019; Kumar et al., 2020). In our early experiments, we found that pre-trained model-based data augmentation does not improve BERT classification performance in full data regime which is consistent with previous work (Kumar et al., 2019). Instead, we use targeted data augmentation, as described in algorithm 1 to improve the robustness of the BERT classifier.

Our data augmentation algorithm is motivated by the fact that BERT relies on the presence of simple markers to distinguish between INFORMATIVE vs UNINFORMATIVE classes. We propose to replace such markers with other words so that model does not rely too much on such markers. We hypothesize that such markers should have high discriminative power since the model relies on these words to distinguish between two categories. In our 


\begin{tabular}{lr}
\hline Model & Mean Dev F1 \\
\hline Bert & $90.46(0.29)$ \\
CT-BERT & $92.72(0.18)$ \\
\hline CT-BERT $_{\text {Aug }}$ & $\mathbf{9 2 . 8 4}(0.26)$ \\
\hline
\end{tabular}

Table 2: Mean F1 with (STD) on Dev partition of the data. Mean and STD are computed over 5 runs.

\begin{tabular}{l}
\hline Top 20 unigrams \\
\hline breaking, bringing, case, cases, confirmed, \\
confirms, county, deaths, department, died, \\
employee, help, new, old, positive, \\
recovered, reported, tested, total, user \\
\hline
\end{tabular}

Table 3: Top 20 unigram features identified using chisquared test statistics

experiments, we use chi-squared stats to find unigrams which have the most discriminative power and use them as markers. Then, we replace such words with $M A S K$ token and use BERT to find the replacement of such words. We generate one example for every example in the training set which doubles the size of our training set. We use CTBERT $_{\text {Aug }}$ to refer to the CT-BERT model trained using augmented data.

\section{Experiments}

For our experiments, we use Huggingface's transformers package (Wolf et al., 2019). We use AdamW optimizer with a learning rate of $4 e-5$, batch size of 32 , and 70 as the max sequence length. We use the pooled representation of the hidden state of the first special token([CLS]) as the sentence embedding. A dropout probability of 0.1 is applied to the sentence embedding before passing it to the 1-layer Softmax classifier. All models are trained for 7 epochs and the best model is selected on the basis of dev set performance.

For the model robustness experiment, we create a modified dev set where we add " 10 deaths" to the beginning of all tweets in the dev set and test model performance against that. Since the main purpose of this paper is not to identify such universal adversarial triggers (Wallace et al., 2019), but to highlight the lack of robustness, our modified dev set provides a proxy for robustness experiment.

\section{Results}

We use train set to train BERT and CT-BERT model and report performance on dev set. Table 2 shows

\begin{tabular}{lr}
\hline Model & Dev F1 \\
\hline CT-BERT & 7.28 \\
CT-BERT $_{\text {Aug }}$ & 34.68 \\
\hline
\end{tabular}

Table 4: F1 score on Dev set where "10 deaths" is prepended to all tweets in devset

the mean F1 performance with STD on dev set. All experiments are repeated 5 times. Since, our task is a tweets classification task, as expected CT-BERT which is pre-trained on tweets data, performs better than the bert-large-uncased BERT model. Given that both models have the same number of parameters and have been trained with the same hyperparameters, the better performance of CT-BERT can be attributed to CT-BERT model pre-training. We submitted CT-BERT predictions on the test set for the final submission, and obtained $89.92 \mathrm{~F} 1$ score on the testset ${ }^{2}$.

Data Augmentation further improves CTBERT's classification performance from 92.72 to 92.84. Table 3 represents the top 20 unigrams identified using chi-squared statistics ${ }^{3}$ (Liu and Setiono, 1995). Most of these words appear more frequently in one category over another and that's why these are ranked higher in chi-squared test.

As shown in Table 4, we observe a sharp drop in CT-BERT performance on dev set when we add " 10 deaths" to all tweets. This sharp drop comes from that fact that model classified most of the uninformative tweets as informative tweets because of the presence of death count information. While data augmentation helps in improving the robustness, CT-BERT $_{\text {Aug }}$ still struggles in adversarial setting.

\section{Discussion}

Pre-trained language models provide state of the art performance on most of the NLP benchmark datasets. Unsurprisingly, CT-BERT does well on the shared task dataset. While these models can be further improved using ensemble techniques, improved fine-tuning (?), a more important research direction is to assess the applicability of such models to identify informative information in real-world applications.

We show the fragile nature of such classifiers using a simple adversarial task where the model

\footnotetext{
${ }^{2}$ For final submission, we submitted a CT-BERT model predictions based on dev set accuracy.

${ }^{3}$ https://scikit-learn.org/stable/ modules/generated/sklearn. feature_ selection.chi2.html
} 
fails to classify an uninformative tweet if we add "10 deaths" to it. While not reported in the paper, we observe similar results for any "N deaths" pattern. On a social media platform such as Twitter, fake news or uninformative tweets might contain a similar pattern, and state of the art classifiers might not be able to correctly identify them. It raises serious concerns about the robustness of such systems. While our proposed data augmentation helps in improving the model robustness, it still falls short from what is expected from a trustworthy system.

\section{Conclusion}

In this paper, we introduce BERT based classifiers to identify informative tweets. We show that while such classifiers performs well on standard test benchmarks, they exploits easy clues for classification and their performance degrades drastically in the presence of simple adversarial triggers. We show that targeted data augmentation can help in improving the robustness and classification performance of such classifiers. In future, we will explore universal adversarial triggers (Song et al., 2020) to create a more challenging adversarial dataset and will also explore other techniques such as stability training (Zheng et al., 2016) to improve model robustness.

\section{References}

Jacob Devlin, Ming-Wei Chang, Kenton Lee, and Kristina Toutanova. 2018. Bert: Pre-training of deep bidirectional transformers for language understanding. arXiv preprint arXiv:1810.04805.

Andre Esteva, Anuprit Kale, Romain Paulus, Kazuma Hashimoto, Wenpeng Yin, Dragomir Radev, and Richard Socher. 2020. Co-search: Covid-19 information retrieval with semantic search, question answering, and abstractive summarization. arXiv preprint arXiv:2006.09595.

Varun Kumar, Ashutosh Choudhary, and Eunah Cho. 2020. Data augmentation using pre-trained transformer models. arXiv preprint arXiv:2003.02245.

Varun Kumar, Hadrien Glaude, Cyprien de Lichy, and William Campbell. 2019. A closer look at feature space data augmentation for few-shot intent classification. arXiv preprint arXiv:1910.04176.

Huan Liu and Rudy Setiono. 1995. Chi2: Feature selection and discretization of numeric attributes. In Proceedings of 7th IEEE International Conference on Tools with Artificial Intelligence, pages 388-391. IEEE.
R Thomas McCoy, Ellie Pavlick, and Tal Linzen. 2019. Right for the wrong reasons: Diagnosing syntactic heuristics in natural language inference. arXiv preprint arXiv:1902.01007.

Martin Müller, Marcel Salathé, and Per E Kummervold. 2020. Covid-twitter-bert: A natural language processing model to analyse covid-19 content on twitter. arXiv preprint arXiv:2005.07503.

Dat Quoc Nguyen, Thanh Vu, Afshin Rahimi, Mai Hoang Dao, Linh The Nguyen, and Long Doan. 2020. WNUT-2020 Task 2: Identification of Informative COVID-19 English Tweets. In Proceedings of the 6th Workshop on Noisy User-generated Text.

Timothy Niven and Hung-Yu Kao. 2019. Probing neural network comprehension of natural language arguments. arXiv preprint arXiv:1907.07355.

Gautam Kishore Shahi, Anne Dirkson, and Tim A Majchrzak. 2020. An exploratory study of covid19 misinformation on twitter. arXiv preprint arXiv:2005.05710.

Liwei Song, Xinwei Yu, Hsuan-Tung Peng, and Karthik Narasimhan. 2020. Universal adversarial attacks with natural triggers for text classification. arXiv preprint arXiv:2005.00174.

Ashish Vaswani, Noam Shazeer, Niki Parmar, Jakob Uszkoreit, Llion Jones, Aidan N Gomez, Łukasz Kaiser, and Illia Polosukhin. 2017. Attention is all you need. In Advances in neural information processing systems, pages 5998-6008.

Eric Wallace, Shi Feng, Nikhil Kandpal, Matt Gardner, and Sameer Singh. 2019. Universal adversarial triggers for attacking and analyzing nlp. arXiv preprint arXiv:1908.07125.

Jason Wei and Kai Zou. 2019. Eda: Easy data augmentation techniques for boosting performance on text classification tasks. arXiv preprint arXiv:1901.11196.

Thomas Wolf, Lysandre Debut, Victor Sanh, Julien Chaumond, Clement Delangue, Anthony Moi, Pierric Cistac, Tim Rault, Rémi Louf, Morgan Funtowicz, et al. 2019. Huggingface's transformers: Stateof-the-art natural language processing. ArXiv, pages arXiv-1910.

Xing Wu, Shangwen Lv, Liangjun Zang, Jizhong Han, and Songlin Hu. 2019. Conditional bert contextual augmentation. In International Conference on Computational Science, pages 84-95. Springer.

Stephan Zheng, Yang Song, Thomas Leung, and Ian Goodfellow. 2016. Improving the robustness of deep neural networks via stability training. In Proceedings of the ieee conference on computer vision and pattern recognition, pages 4480-4488. 